\title{
Calculations of Triple Differential Cross-Sections for the Ionization of H (3S) by Electron Impact
}

\author{
Tania Noor ${ }^{1}$, Farhana Shirin Chowdhury ${ }^{2}$ \\ ${ }^{1}$ Department of Mathematics, Premier University, Chittagong, Bangladesh \\ ${ }^{2}$ Department of Computer Science and Engineering, Premier University, Chittagong, Bangladesh
}

Email address:

taniatima@gmail.com (T. Noor), fshirin2007@gmail.com (F. S. Chowdhury)

\section{To cite this article:}

Tania Noor, Farhana Shirin Chowdhury. Calculations of Triple Differential Cross-Sections for the Ionization of H (3S) by Electron Impact. American Journal of Modern Physics. Vol. 5, No. 6, 2016, pp. 162-171. doi: 10.11648/j.ajmp.20160506.11

Received: September 16, 2016; Accepted: September 26, 2016; Published: October 15, 2016

\begin{abstract}
In the present study we have calculated the Triple Differential Cross-Sections (TDCS) for the ionization of metastable 3S-state hydrogen atoms at $250 \mathrm{eV}$ incidence electron energy for various kinematic conditions in the asymmetric coplanar geometry. A final state wave function of multiple scattering theory [11] has been used in the present study. The present results show a good qualitative agreement with the existing hydrogenic ground state experimental data and various related theoretical results. Some results of hydrogenic metastable states are also presented here for comparisons. However there is no existing theoretical and experimental works on hydrogenic metastable 3S-state except an existing theoretical first born result of Vučič et al. [19].
\end{abstract}

Keywords: Electron, Cross-Section, Ionization, Scattering

\section{Introduction}

The ionization study of atoms by electrons and positrons is one of the most important challenges problems for astrophysics, plasma physics and radiation physics. In quantum mechanical way Bethe [1] was first treated the atomic ionization by fast particles. Over the last four decades the study of triple differential cross-sections (TDCS) in electron-hydrogen atom ionization collisions has become progressively interesting. The atomic hydrogen ionization by fast particles is a good form for perturbation theory due to the availability of experimental results [2-6]. Such electronelectron coincidence experiments called (e, 2e) experiments [5] which gives a clear knowledge of kinematics of collision by giving evidence about the direction of scattered and ejected electrons. The Triple differential cross-sections (TDCS) is proportional to the quality measured of this kind of experiment. The (e, 2e) experiment on atomic hydrogen was firstly done at low incident energy [2]. The first absolute Triple differential cross-sections (TDCS) for asymmetric geometries was established by Ehrhardt et al [4]. Many researchers have been successfully investigated such experiments on ionization process both in ground states [7-
17] and metastable states [18-28] of atomic hydrogen by electron and positron impact.

Using the (e, 2e) experiment, the study of Triple differential cross-sections (TDCS) were widely studied for ground state hydrogen atoms both theoretically [29-31] and experimentally [32]. Whereas no experimental works on Triple differential cross-sections (TDCS) for the ionization of hydrogenic metastable states, have been done yet. While the total cross sections [TCS] measurement was done experimentally much earlier [33-34].

The first theoretical study on the second born triple differential cross-sections (TDCS) for the coplanar asymmetric ionization of hydrogenic metastable 2S-state by fast electrons was done by Vučičč et al. [19]. Vučič et al. [19] has also been calculated the first born TDCS at $5 \mathrm{eV}$ ejected electron energy for the ionization of $\mathrm{H}(3 \mathrm{~S})$ by $250 \mathrm{eV}$ incident electron energy. After this successful study the corrected double continuum BBK wave function [31] have been shown by Hafid et al. [21].

Our present study is on the ionization of metastable 3Sstate hydrogen atoms by $250 \mathrm{eV}$ incident energy triple 
differential cross-sections (TDCS) has been calculated following a multiple scattering theory of Das and Seal [11]. Using this multiple scattering theory [11] good cross sectional results for the ground state hydrogenic ionization $[11,13]$ and hydrogenic metastable state ionization $[22,23$, $40,41]$ by electrons have been calculated successfully. This multiple scattering theory [11] is also used widely in the study of medium-heavy atoms ionization by electrons and positrons in the relativistic energy region $[17,24,35,36]$. So the present study will also be interesting using this multiple scattering theory [11].

\section{Theory}

Atomic hydrogen ionization by electrons in most detailed form are presently available of following type

$$
e^{-}+H(3 S) \rightarrow H^{+}+2 e^{-}
$$

Here $3 \mathrm{~S}$ denotes the metastable state of the present target, obtained by analyzing triple differential cross sections (TDCS) in the coplanar geometry and measured in (e, 2e) coincidence experiments. The experiment where the ejected electron is detected in coincidence with the scattered electron is considered here as (e, 2e) experiment [5]. In an (e, 2e) reaction an incident electron of momentum $\bar{p}_{l}$ and energy $E_{i}$ will produce on collision with the target two electrons having energies $E_{1}$ and $E_{2}$ and momentum $\overline{p_{1}}$ and $\overline{p_{2}}$ triple differentia; cross sections (TDCS) is a measure of the probability that emitted respectively into the solid angles $d \Omega_{1}$ and $d \Omega_{2}$ centered about the directions $\left(\theta_{1}, \Phi_{1}\right)$ and $\left(\theta_{2}, \Phi_{2}\right)$.

The symbol $\frac{d^{3} \sigma}{d \Omega_{1} d \Omega_{2} d E_{1}}$ is generally used for TDCS. For unpolarized incident electrons and targets, it is a function of the quantities $E_{i}, E_{1}$ or $E_{2}, \theta_{1}, \theta_{2}$ and $\Phi=\Phi_{1}+\Phi_{2}$. After integrating the triple differential cross sections (TDCS) over $d \Omega_{1}, d \Omega_{2}$ or $d E_{2}$ we can form various double and single differential cross sections. The present result will help us to calculate the same in near future. The total differential cross section is obtained by integrating over all outgoing scattering angles and energies, and depends only in the incident energy $E_{i}$. For the theoretical analysis of the collision, it is valuable when reading $(\mathrm{e}, 2 \mathrm{e})$ coincidence experiments to distinguish between several kinematical arrangements. The (e, 2e) coincidence experiments have important insinuation for the theoretical analysis of the collision. The momentum $\overline{p_{l}}, \overline{p_{1}}$ and $\overline{p_{2}}$ are in the same plane because of the first distinction can be made between coplanar geometries whereas the non-coplanar geometries the momentum $\overline{p_{2}}$ is out of the $\left(\overline{p_{l}} \cdot \overline{p_{1}}\right)$ reference plane. For asymmetric and symmetric geometries there is another distinction. A fast electron energy $E_{i}$ is incident on the target atom and a fast scattered electron is detected in coincidence with a slow ejected electron, in asymmetric geometries. Ehrhardt et al. [5] first performed this experiment. Instead, symmetric geometries are derived by the condition that $\theta_{1} \cong$ $\theta_{2}$ and $E_{1} \cong E_{2}$. The (e, 2e) symmetric coincidence experiments by Amaldi et al. [37] have been monitored by this type of experiments.

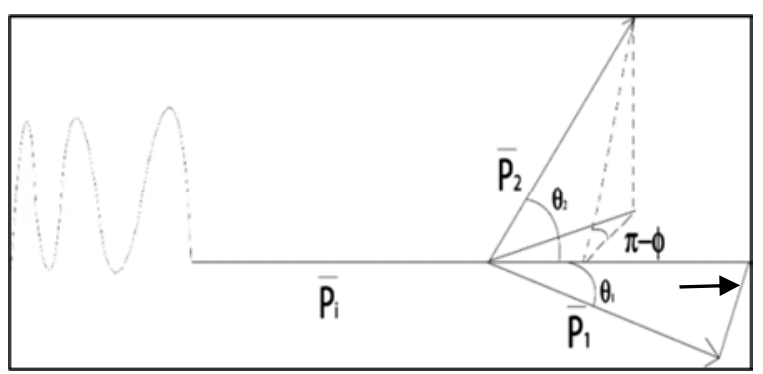

The multiple scattering theory of ionization by electrons of hydrogen atoms is defined in detail in [11]. The T-matrix element for ionization of hydrogen atoms by electrons [11] is given by

$$
T_{f i}=\left\langle\Psi_{f}^{(-)}\left(\bar{r}_{1}, \bar{r}_{2}\right)\left|V_{i}\left(\bar{r}_{1}, \bar{r}_{2}\right)\right| \Phi_{i}\left(\bar{r}_{1}, \bar{r}_{2}\right)\right\rangle,
$$

Here the perturbation potential $V_{i}\left(\bar{r}_{1}, \bar{r}_{2}\right)$ is given by

$$
V_{i}\left(\bar{r}_{1}, \bar{r}_{2}\right)=\frac{1}{r_{12}}-\frac{Z}{r_{2}}
$$

For hydrogen atom nuclear charge $(\mathrm{Z})=1, r_{1}$ and $r_{2}$ are the distance of the two electrons from the nucleus and $r_{12}$ is the distance between the two electrons.

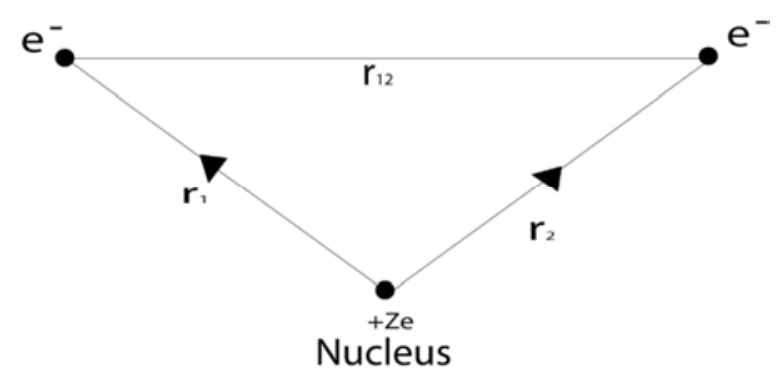

The initial channel unperturbed wave function is,

$$
\Phi_{i}\left(\bar{r}_{1}, \bar{r}_{2}\right)=\frac{e^{i \cdot \bar{p}_{i} \cdot \bar{r}_{2}}}{(3 \pi)^{3 / 2}} \varphi_{3 S}\left(\bar{r}_{1}\right)=\frac{e^{i \cdot \bar{p}_{i} \cdot \bar{r}_{2}}}{(3 \pi)^{3 / 2}} \cdot \frac{1}{81 \sqrt{3 \pi}}\left(27-18 \bar{r}_{1}+2 \bar{r}_{1}^{2}\right) e^{-\lambda_{1} r_{1}}
$$

Where

$$
\varphi_{3 S}\left(\bar{r}_{1}\right)=\frac{1}{81 \sqrt{3 \pi}}\left(27-18 \bar{r}_{1}+2 \bar{r}_{1}^{2}\right) e^{-\lambda_{1} r_{1}}
$$

and

$$
\lambda_{1}=1 / 3
$$

is the incident electron momentum, $\Psi_{f}^{(-)}\left(\bar{r}_{1}, \bar{r}_{2}\right)$ and is the final three-particle scattering state wave function with the electrons being in the continuum with momenta $\bar{p}_{1}, \bar{p}_{2}$. Coordinates of the two electrons taken to be $\bar{r}_{1}$ and $\bar{r}_{2}$.

Here $\Psi_{f}^{(-)}\left(\bar{r}_{1}, \bar{r}_{2}\right)$ is approximate wave function [11] is given by

Equation (5) is the hydrogenic $3 \mathrm{~S}$-state wave function, $\bar{p}_{1}$ 


$$
\Psi_{f}^{(-)}\left(\overline{r_{1}}, \overline{r_{2}}\right)=N\left(\overline{p_{1}}, \overline{p_{2}}\right)\left[\phi_{\overline{p_{1}}}^{(-)}\left(\overline{r_{1}}\right) e^{i \overline{\overline{p_{2}} \cdot \overline{r_{2}}}}+\phi_{\overline{p_{2}}}^{(-)}\left(\overline{r_{2}}\right) e^{i \overline{p_{1}} \cdot \overline{r_{1}}}+\phi_{\bar{p}}^{(-)}(\bar{r}) e^{i \bar{P} \cdot \bar{R}}-2 e^{i \overline{p_{1}} \cdot \overline{r_{1}}+i \overline{p_{2}} \cdot \overline{r_{2}}}\right] /(3 \pi)^{3}
$$

Where

$$
\bar{r}=\frac{\overline{r_{1}}-\bar{r}_{2}}{2}, \bar{R}=\left(\bar{r}_{1}+\bar{r}_{2}\right) / 2, \bar{p}=\left(\overline{p_{2}}-\overline{p_{1}}\right), \bar{P}=\overline{p_{2}}+\overline{p_{1}}
$$

The scattering amplitude [11] may be written as

$$
f\left(\bar{p}_{1}, \bar{p}_{2}\right)=N\left(\bar{p}_{1}, \bar{p}_{2}\right)\left[f_{e T}+f_{P T}+f_{P e}-2 f_{P W B}\right]
$$

Where $f_{e T}, f_{P T}, f_{P e}$ and $f_{P W B}$ are the amplitudes corresponding to the four terms of equation (6) respectively. The normalization constant $N\left(\bar{p}_{1}, \bar{p}_{2}\right)$ is given by

$$
\left|N\left(\bar{p}_{1}, \bar{p}_{2}\right)\right|^{-2}=\left|7-2\left[\lambda_{1}+\lambda_{2}+\lambda_{3}\right]-\left[\frac{2}{\lambda_{1}}+\frac{2}{\lambda_{2}}+\frac{2}{\lambda_{3}}\right]+\left[\frac{\lambda_{1}}{\lambda_{2}}+\frac{\lambda_{1}}{\lambda_{3}}+\frac{\lambda_{2}}{\lambda_{1}}+\frac{\lambda_{2}}{\lambda_{3}}+\frac{\lambda_{3}}{\lambda_{1}}+\frac{\lambda_{3}}{\lambda_{2}}\right]\right|
$$

Where

(2) we get,

$$
\begin{aligned}
& \lambda_{1}=e^{\frac{\pi \alpha_{1}}{2}} \Gamma\left(1-i \alpha_{1}\right), \alpha_{1}=\frac{1}{p_{1}} \\
& \lambda_{2}=e^{\frac{\pi \alpha_{2}}{2}} \Gamma\left(1-i \alpha_{2}\right), \alpha_{2}=\frac{1}{p_{2}} \\
& \lambda_{3}=e^{\frac{\pi \alpha}{2}} \Gamma(1-i \alpha), \alpha=-\frac{1}{p}
\end{aligned}
$$

$$
T_{f i}=T_{B}+T_{B^{\prime}}+T_{i}-2 T_{P B}
$$

Where

$$
\begin{gathered}
T_{B}=\left\langle\Phi_{\bar{p}_{1}}^{(-)}\left(\overline{r_{1}}\right) e^{i \bar{p}_{2} \cdot r_{2}}\left|V_{i}\right| \Phi_{i}\left(\bar{r}_{1}, \bar{r}_{2}\right)\right\rangle \\
T_{B^{\prime}}=\left\langle\Phi_{p 2}^{(-)}\left(\bar{r}_{2}\right) e^{i \bar{p}_{1} \cdot r_{1}}\left|V_{i}\right| \Phi_{i}\left(\bar{r}_{1}, \bar{r}_{2}\right)\right\rangle \\
T_{i}=\left\langle\Phi_{\bar{p}}^{(-)}(\bar{r}) e^{i \cdot \bar{P} \cdot \bar{R}}\left|V_{i}\right| \Phi_{i}\left(\bar{r}_{1}, \bar{r}_{2}\right)\right\rangle \\
T_{P B}=\left\langle e^{i \bar{p}_{1} \cdot \bar{r}_{1}+i \bar{p}_{2} \cdot \bar{r}_{2}}\left|V_{i}\right| \Phi_{1}\left(\bar{r}_{1}, \bar{r}_{2}\right)\right\rangle
\end{gathered}
$$

$\phi_{q}^{(-)}(\bar{r})=e^{\frac{\pi \alpha}{2}} \Gamma(1+i \alpha) e^{i q \cdot \bar{r}}{ }_{1} F_{1}(-i \alpha, 1,-i[q r+\bar{q} \cdot \bar{r}])$

Where $\alpha_{1}=\frac{1}{p_{1}}$ for $\bar{q}=\bar{p}, \alpha_{2}=\frac{1}{p_{2}}$ for $\bar{q}=\overline{p_{2}}$ and $\alpha=-\frac{1}{p}$ for

Here equation (10) is called first born term that can be written as $\bar{q}=\bar{p}$

Now applying equations (3), (4), (5) and (6) in equation

$$
T_{B}=\frac{1}{81(3 \pi)^{2}}\left\langle\Phi_{\bar{p}_{1}}^{(-)}\left(\overline{r_{1}}\right) e^{i \bar{p}_{2} \cdot r_{2}}\left|\frac{1}{r_{12}}-\frac{1}{r_{2}}\right| e^{i \cdot \bar{p}_{i} \cdot \bar{r}_{2}}\left(27-18 r_{1}+2 r_{1}^{2}\right) e^{-\lambda_{1} \cdot r_{1}}\right\rangle
$$

With

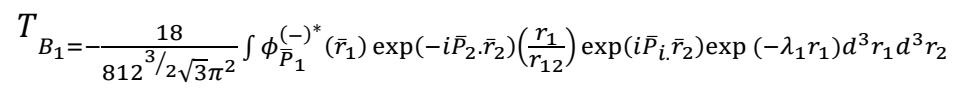

$$
\begin{aligned}
& T_{B_{2}}=\frac{18}{812^{3 / 2 \sqrt{3} \pi^{2}}} \int \phi_{\bar{P}_{1}}^{(-)^{*}}{ }^{*}\left(\bar{r}_{1}\right) \exp \left(-i \bar{P}_{2} \cdot \bar{r}_{2}\right)\left(\frac{r_{1}}{r_{2}}\right) \exp \left(i \bar{P}_{i} \cdot \bar{r}_{2}\right) \exp \left(-\lambda_{1} r_{1}\right) d^{3} r_{1} d^{3} r_{2}
\end{aligned}
$$

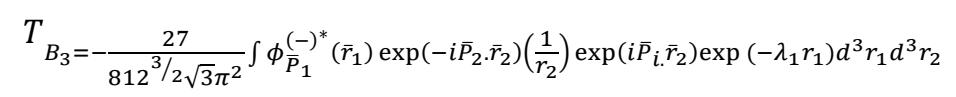

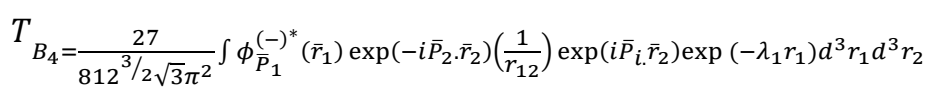

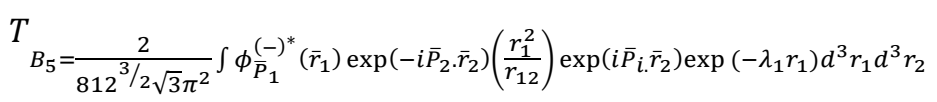

$$
\begin{aligned}
& T{ }_{B_{6}}=\frac{2}{812^{3 / 2 \sqrt{3} \pi^{2}}} \int \phi_{\bar{P}_{1}}^{(-)_{1}^{*}}\left(\bar{r}_{1}\right) \exp \left(-i \bar{P}_{2} \cdot \bar{r}_{2}\right)\left(\frac{r_{1}^{2}}{r_{2}}\right) \exp \left(i \bar{P}_{i} \cdot \bar{r}_{2}\right) \exp \left(-\lambda_{1} r_{1}\right) d^{3} r_{1} d^{3} r_{2}
\end{aligned}
$$

After analytical calculations using Lewis integral [38], we have

$$
T_{B 1}=\frac{16 \sqrt{2} \exp \left(\frac{\pi \alpha_{1}}{2}\right)\left[\left(1-i \alpha_{1}\right)\left(\overline{t^{2}}-\overline{p_{1}} \cdot \bar{t}-i \alpha_{1} \overline{p_{1}} \cdot \bar{t}\right) \exp \left(i \alpha_{1} \ln \vartheta\right)\right.}{3^{\frac{5}{2}} t^{2}\left\{\overline{t^{2}}-\left(i+\overline{p_{1}}\right)^{2}\right\}\left\{1+\left(\bar{t}-\overline{p_{1}}\right)^{2}\right\}^{2}}
$$

Where 


$$
\vartheta=\frac{1+\left(\bar{t}-\overline{p_{1}}\right)^{2}}{\overline{t^{2}}-\left(i+\overline{p_{1}}\right)^{2}}
$$

With

$$
\bar{t}=\overline{p_{l}}-\overline{p_{2}}
$$

Similarly for our present second born results we calculated analytically the above equations (11), (12) and (13) using Lewis integral [38]. After that we computed the above equations numerically using the Gaussian quadrature formula. The triple differential cross sections for direct amplitude is finally calculated by

$$
\frac{d^{3} \sigma}{d \Omega_{1} d \Omega_{2} d E_{1}}=\frac{p_{1} p_{2}}{p_{i}}\left|T_{f i}\right|^{2}
$$

Where $E_{1}$ is the energy of the ejected electron. Hence in our present work we have calculated and computed the TDCS of equation (16).

\section{Result and Discussion}

We have investigated here the ionization of hydrogenic metastable 3S-state by incident electrons. We have calculated in this section the triple differential cross-sections (TDCS) at $E_{i}=250 \mathrm{eV}$ incident energy with the ejected electron energy of $5 \mathrm{eV}$. Electron-hydrogen ionization from ground state theoretical results of Dal et al. [25], the BBK model of Brauner et al. [31] and the experimental results of Ehrhardt et al. [5] are presented here for comparisons. We also compared our present results with the earlier works of hydrogenic $2 \mathrm{~S}$ state [12] ionization result and recent works on hydrogenic 2P-state [39] ionization results. We have completed the triple differential cross-section (TDCS) results of the present calculation and the first born calculation of equation (1). Our present first born result also compared with the first born result of Vučič et al. [19]. We studied here the second born triple differential cross sections for the ionization of metastable 3S-state hydrogen atoms by electrons at incident electron energy of $250 \mathrm{eV}$ for some varied ejected angles $\left(\theta_{1}\right)$ and for a fixed scattering angle $\left(\theta_{2}\right)$. The following ten graphs gives a glimpse of our calculated results. In the following figures we have designed and displayed the electron impact triple differential cross sections (TDCS) changing against the ejection angle $\left(\theta_{1}\right)$ of the ejected electron. The recoil position of the figures are directed to the region where $\theta_{1}\left(0^{0}-150^{\circ}\right)$ and $\Phi=0^{0}$ on other hand the binary region is from $\theta_{1}\left(150^{\circ}-360^{\circ}\right)$ and $\Phi=180^{\circ}$.

We have considered here the triple differential crosssections (TDCS) for the ionization of metastable 3S-state hydrogen atoms by electrons for the incident electron energy of $E_{i}=250 \mathrm{eV}$ and ejected electron energies $E_{1}=5 \mathrm{eV}$ and $50 \mathrm{eV}$. We calculated the TDCS for various scattering angles like $\theta_{2}=3^{0}$ (Fig-1), $15^{0}$ (Fig-2), $25^{0}$ (Fig-3), $5^{0}$ (Fig-4), $7^{0}$ (Fig-5), $9^{0}$ (Fig-6), $11^{0}$ (Fig-7), $15^{0}$ (Fig-8) and20 (Fig-9). In the last figure (Fig-10) we compared our result with $\mathrm{Vu} \check{c}$ i $\check{c}$ et al. [19]. The continuum state of the atomic hydrogen is the final state scattering wave function $\Psi_{f}^{(-)}\left(\bar{r}_{1}, \bar{r}_{2}\right)$. According to the previous study on metastable 2P- state [39] hydrogen atoms by electrons, the final continuum state shows a rise in the recoil lobe amplitude. In our present study the final continuum state of metastable 3Sstate hydrogen atoms by electrons shows a fall in the binary region whether it shows a prominent rise in the recoil region amplitude. Comparing with other results of $2 \mathrm{~S}$ and $2 \mathrm{P}$-state hydrogen atoms and with the present 3S- state first born result, the present result shows a large amplitude in the recoil lobe position. After analyzing the previous results it is obvious that the present study play a vital role in the ionization of atomic hydrogen for 3S- state kinematical condition for a large incident energy.

We compared our theoretical results to the well-known BBK model [31] and followed the recommendation of Jones and Madison [26]. We reproduced all the data by a scaling factor of 0.88 for $\theta_{2}=3^{0}$. For the scattering angle $\theta_{2}=$ $15^{0}$ and $25^{\circ}$ we multiplied the experimental data with 0.00224 .

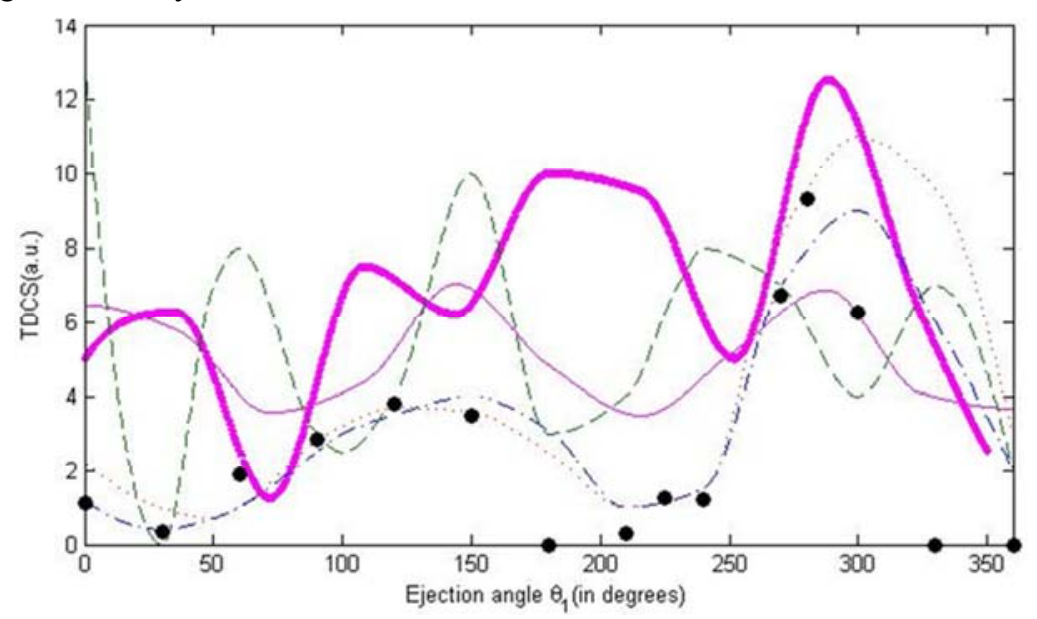

Fig. 1. Triple differential cross sections (TDCS) for ionization of atomic hydrogen by $250 \mathrm{eV}$ electron impact for $\theta_{2}=3^{0}$ vary against the ejected electron $\theta_{1}$ relative to the incident electron direction. The ejected electron energy is $E_{1}=5 \mathrm{eV}$. Theory: Full filled double curve reflects the present calculation. Full single curve represent the first born $3 S$ state result [42]. Dash curve shows the second born $2 P$ state result [39]. Short dash curve focuses hydrogenic ground state second born result [25]; Dash-dotted curve shows hydrogenic ground state BBK model [31] and filled round shows hydrogenic ground state experiments [5] (multiplied by 0.88). 


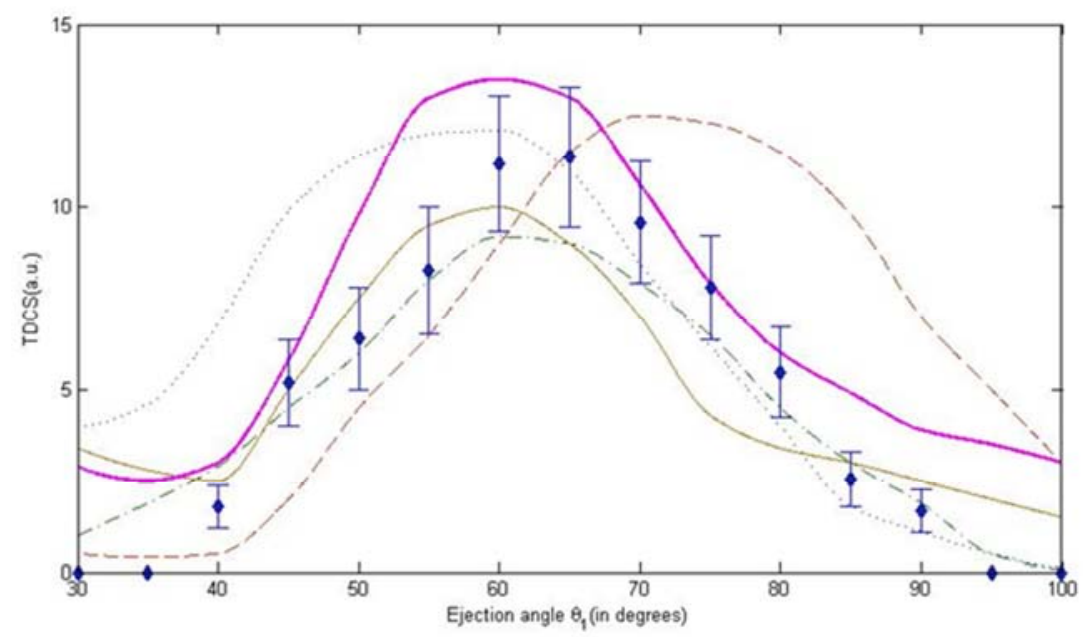

Fig. 2. Triple differential cross sections (TDCS) for ionization of atomic hydrogen by $250 \mathrm{eV}$ electron impact for $\theta_{2}=15^{0}$ vary against the ejected electron $\theta_{1}$ relative to the incident electron direction. The ejected electron energy is $E_{1}=50 \mathrm{eV}$. Theory: Full curve $(\mathrm{m})$ reflects the present calculation. Full single curve (red) represent the first born $3 S$ state result [42]. Dash curve shows the second born 2P state result [39]. Short dash curve focuses hydrogenic ground state second born result [25]; Dash-dotted curve shows hydrogenic ground state BBK model [31], dash-dotted curve reflects the second born 2S-state result [12] and square shows hydrogenic ground state experiments [5] (multiplied by 0.00224).

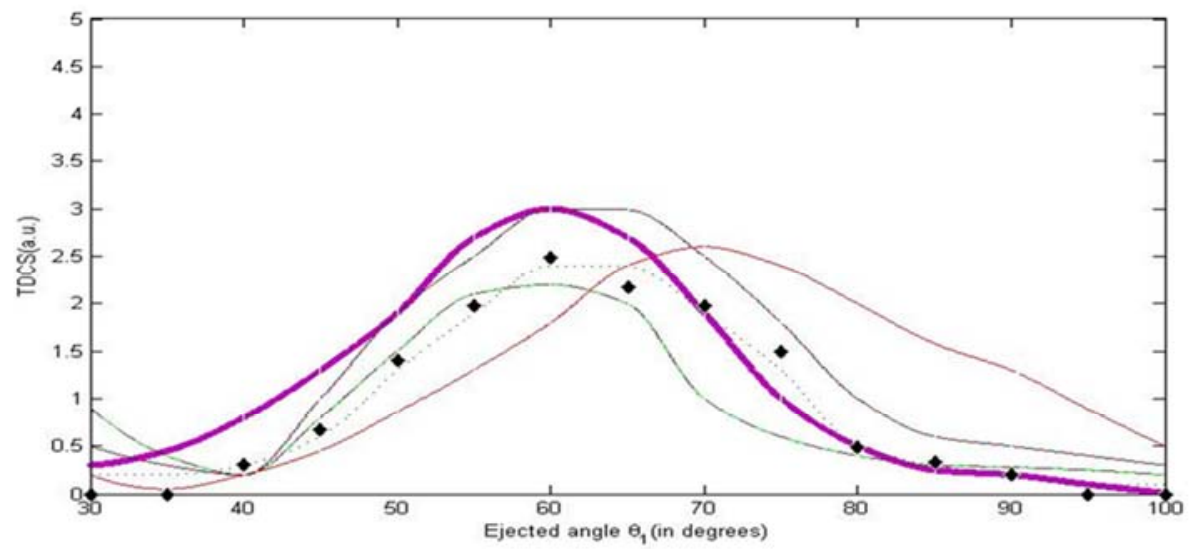

Fig. 3. Triple differential cross sections (TDCS) for ionization of atomic hydrogen by $250 \mathrm{eV}$ electron impact for $\theta_{2}=25^{0}$ vary against the ejected electron $\theta_{1}$ relative to the incident electron direction. The ejected electron energy is $E_{1}=50 \mathrm{eV}$. Theory: Full filled double curve reflects the present calculation. Full single curve represent the first born $3 S$ state result [42]. Dash curve shows the second born 2P state result [39]. Short dash curve focuses hydrogenic ground state second born result [25]; Dash curve shows hydrogenic ground state BBK model [31],dash-dotted curve shows the second born 2S-state result [12] and filled round shows hydrogenic ground state experiments [5] (multiplied by 0.00224).

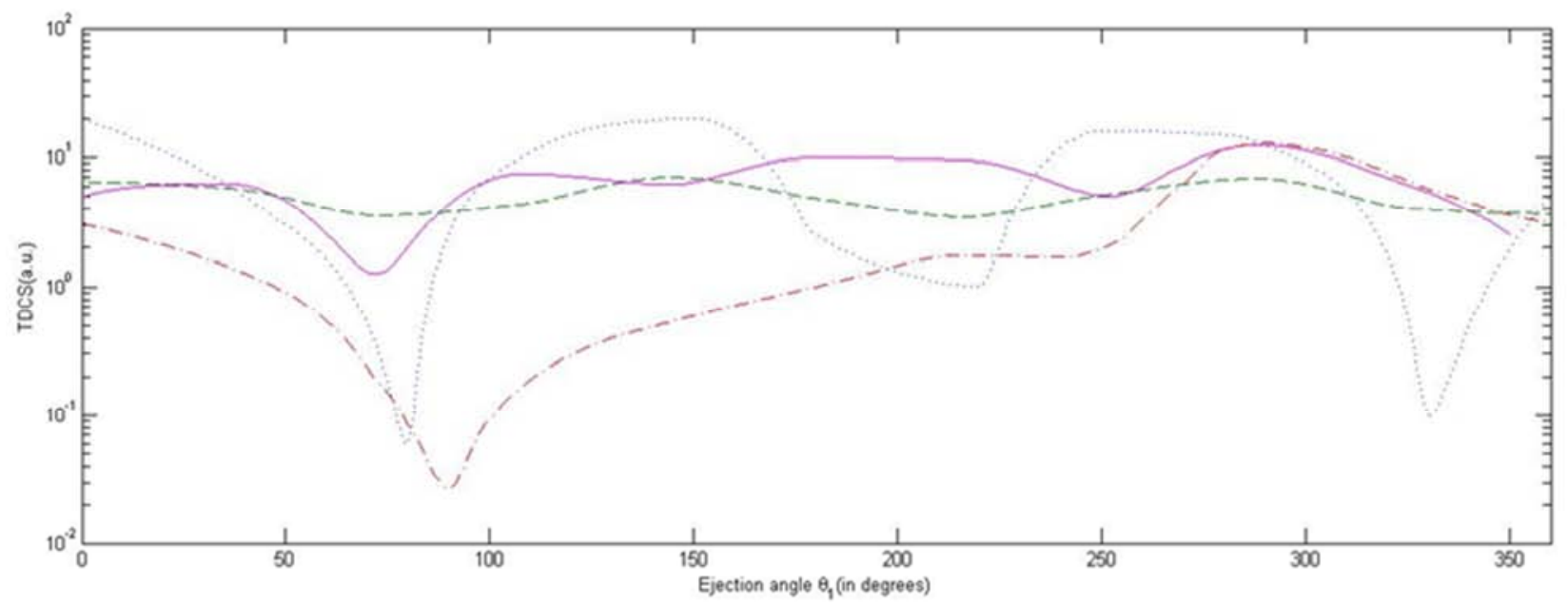

Fig. 4. Triple differential cross sections (TDCS) for ionization of atomic hydrogen by $250 \mathrm{eV}$ electron impact for $\theta_{2}=5^{0}$ vary against the ejected electron $\theta_{1}$ relative to the incident electron direction. The ejected electron energy is $E_{1}=5 \mathrm{eV}$. Theory: Full curve reflects the present calculation. Dash curve represent the first born $3 S$-state result [42]. Short dash curve shows the second born 2P-state result [39].dash-dot curve exhibits the hydrogenic 2S-state result [12]. 


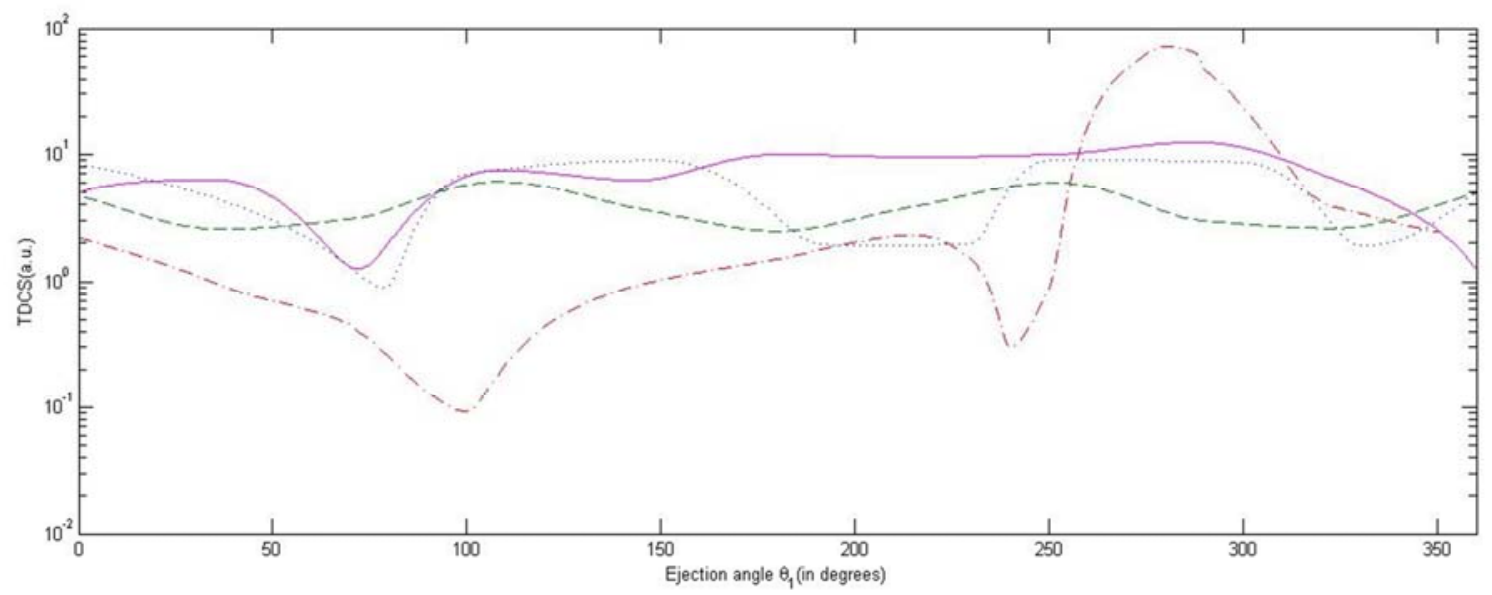

Fig. 5. Triple differential cross sections (TDCS) for ionization of atomic hydrogen by $250 \mathrm{eV}$ electron impact for $\theta_{2}=7^{0}$ vary against the ejected electron $\theta_{1}$ relative to the incident electron direction. The ejected electron energy is $E_{1}=5 \mathrm{eV}$. Theory: Full curve reflects the present calculation. Dash curve represent the first born 3S-state result [42]. Short dash curve shows the second born 2P-state result [39].dash-dot curve exhibits the hydrogenic 2S-state result [12].

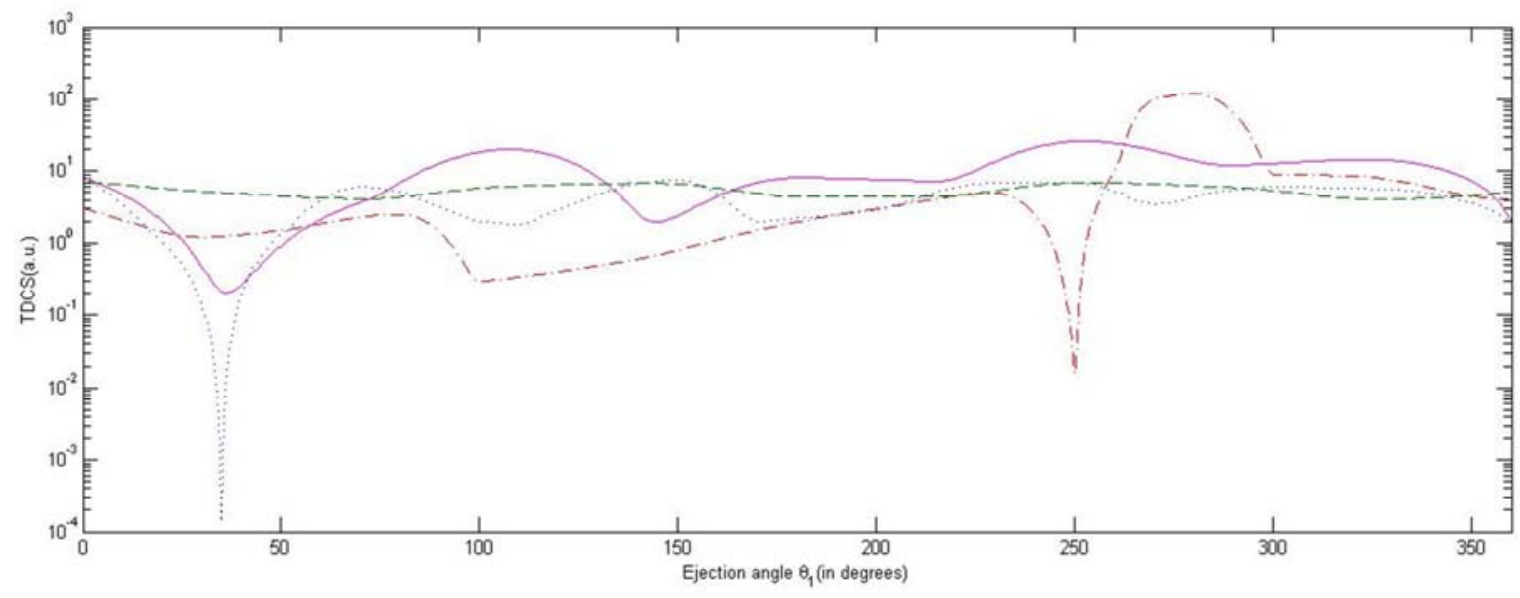

Fig. 6. Triple differential cross sections (TDCS) for ionization of atomic hydrogen by $250 \mathrm{eV}$ electron impact for $\theta_{2}=9^{0}$ vary against the ejected electron $\theta_{1}$ relative to the incident electron direction. The ejected electron energy is $E_{1}=5 \mathrm{eV}$. Theory: Full curve reflects the present calculation. Dash curve represent the first born 3S- state result [42]. Short dash curve shows the second born 2P-state result [39].dash-dot curve exhibits the hydrogenic 2S-state result [12].

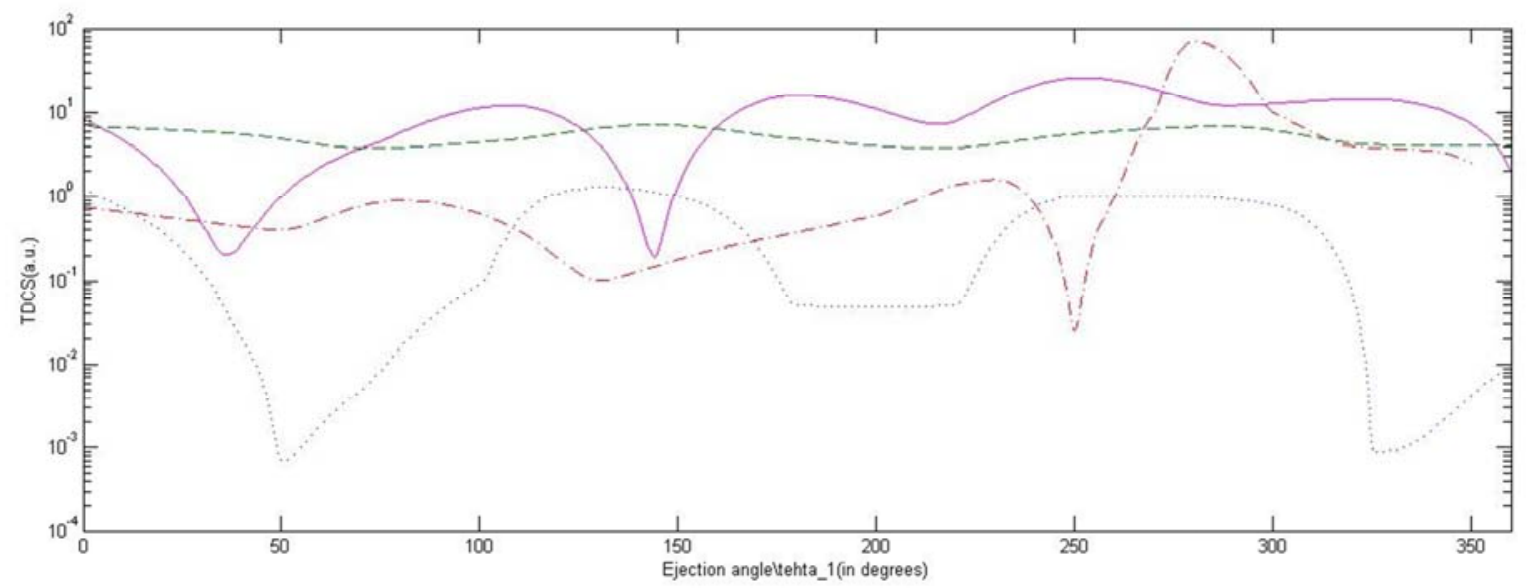

Fig. 7. Triple differential cross sections (TDCS) for ionization of atomic hydrogen by $250 \mathrm{eV}$ electron impact for $\theta_{2}=11^{0}$ vary against the ejected electron $\theta_{1}$ relative to the incident electron direction. The ejected electron energy is $E_{1}=5 \mathrm{eV}$. Theory: Full curve reflects the present calculation. Dash curve represent the first born 3S- state result [42]. Short dash curve shows the second born 2P-state result [39].dash-dot curve exhibits the hydrogenic 2S-state result [12]. 


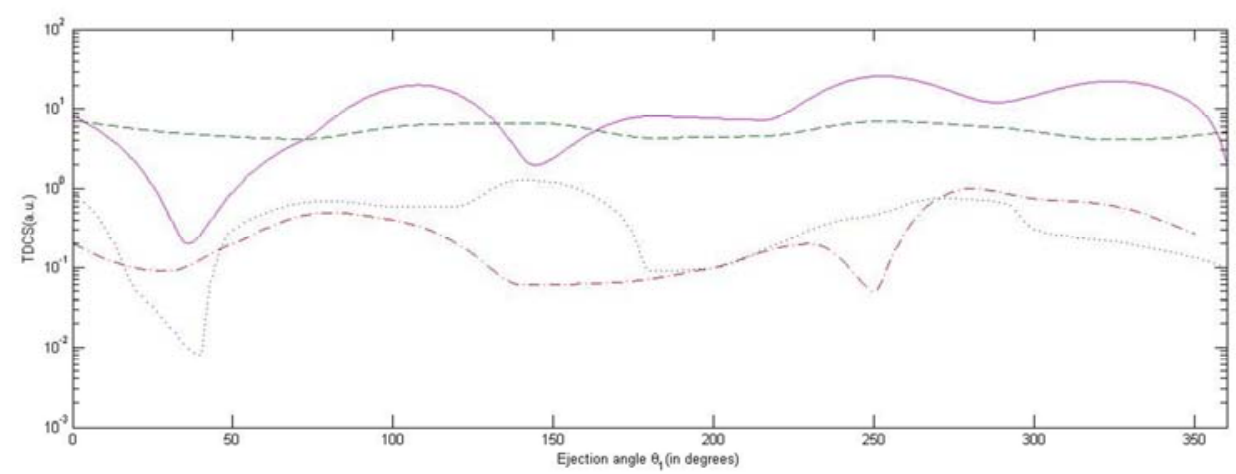

Fig. 8. Triple differential cross sections (TDCS) for ionization of atomic hydrogen by $250 \mathrm{eV}$ electron impact for $\theta_{2}=15^{0}$ vary against the ejected electron $\theta_{1}$ relative to the incident electron direction. The ejected electron energy is $E_{1}=5 \mathrm{eV}$. Theory: Full curve reflects the present calculation. Dash curve represent the first born 3S- state result [42]. Short dash curve shows the second born 2P-state result [39].dash-dot curve exhibits the hydrogenic 2S-state result [12].

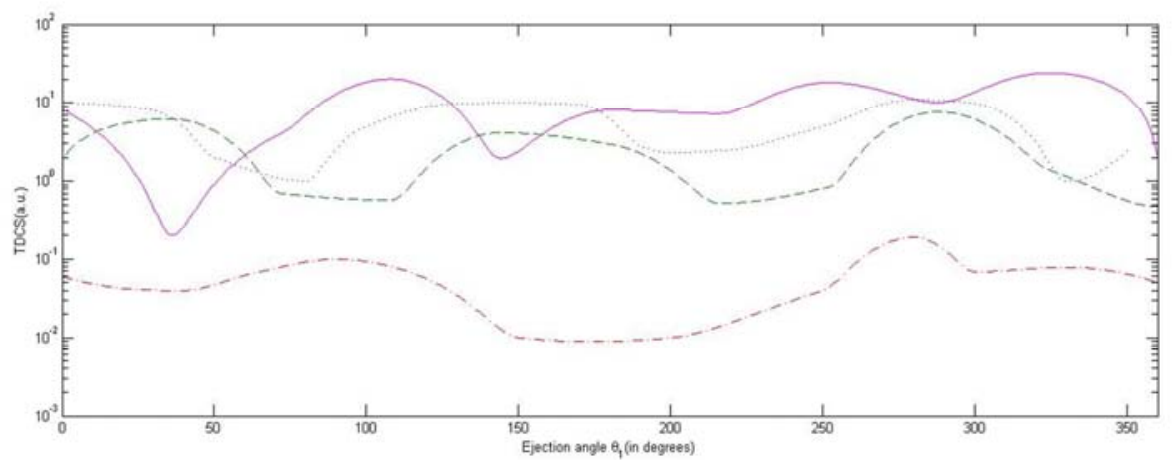

Fig. 9. Triple differential cross sections (TDCS) for ionization of atomic hydrogen by $250 \mathrm{eV}$ electron impact for $\theta_{2}=20^{\circ}$ vary against the ejected electron $\theta_{1}$ relative to the incident electron direction. The ejected electron energy is $E_{1}=5 \mathrm{eV}$. Theory: Full curve reflects the present calculation. Dash curve represent the first born 3S-state result [42]. Short dash curve shows the second born 2P-state result [39].dash-dot curve exhibits the hydrogenic 2S-state result [12].

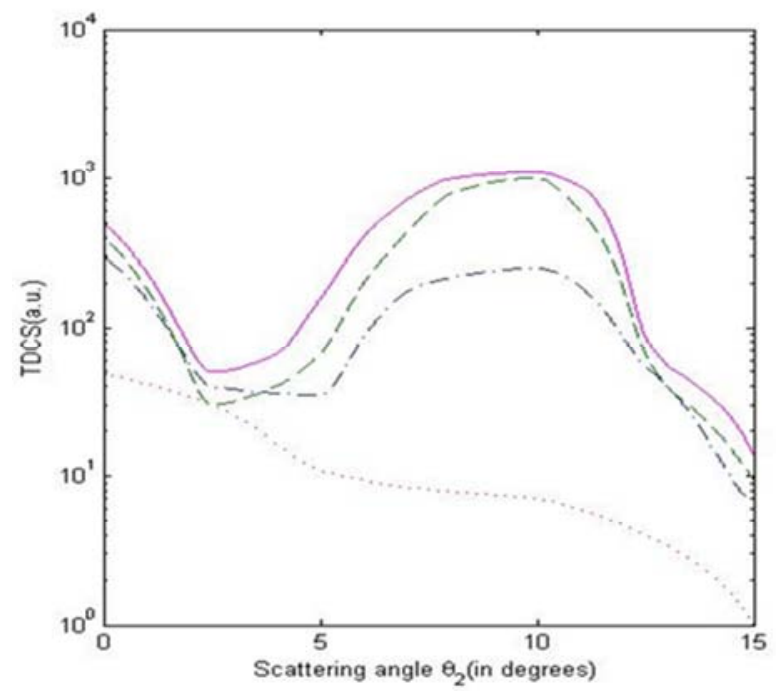

Fig. 10. The first born Triple differential cross sections (TDCS) for ionization of atomic hydrogen by $250 \mathrm{eV}$ electron impact for $\theta_{1}=8.1^{0}$ vary against the scattered electron $\theta_{2}$ relative to the incident electron direction. The ejected electron energy is $E_{1}=5 \mathrm{eV}$. Theory: Full curve reflects the present calculation. Dash curve represent the $H(3 S)$ result [19]. Short dash curve shows the H (1S) [19] result.dash-dot curve exhibits the hydrogenic 2S-state result [19].

Here Fig. 1 the present result shows a comparison with the present first born result [42], the hydrogenic ground state result of BBK model [31], the second born approximation [25], the experimental data [5] and the second born experiment of 2P-state [39] hydrogen atom. Here in our present study the binary peak shows qualitatively good agreement with the BBK model [31], second born result of Dal et al. [25] and experimental data [5]. In the recoil region for both present and first born result shows a fall and a peak. Whereas for the present study the peak in the recoil region are about double to those of the BBK model [31], the second born result [25] and the experimental data [5]. For the 
scattered angle $\theta_{2}=3^{0}$ the binary peak is lowest for our first born result whereas the value is highest for our present result among all theoretical hydrogenic ground state calculations [25] and a good contract shows for the experimental data [5]. In the present study the binary peak shows a slight high position than the other cases.

In Fig. 2 We calculated the triple differential cross section for metastable 3S-state hydrogen atoms by electrons for ejected electron energy $E_{i}=250 \mathrm{eV}$, scattered electron energy $E_{1}=50 \mathrm{eV}$ and scattered angle $\theta_{2}=15^{\circ}$. In this figure we compared our result with hydrogenic ground state second born results [25], hydrogenic ground state BBK results [31] and with the experimental data [5]. We also included here our first born result [42], second born 2S-state and $2 \mathrm{P}$-state result. In this case our first born result for $3 \mathrm{~S}$ state hydrogen atom shows a similar conduct with the hydrogenic ground state BBK results [31] while our present result shows a good approximation with second born hydrogenic ground state result [25].

In a similar way Fig. 3 shows us the $3 \mathrm{~S}$ metastable state for ejected electron energy $E_{i}=250 \mathrm{eV}$, scattered electron energy $E_{1}=50 \mathrm{eV}$ and scattered angle $\theta_{2}=25^{\circ}$. As we increase our scattering angle $\left(\theta_{2}\right)$ the peak of our present study shows a similar agreement with hydrogenic ground state second born results [25]. The figure shows that for our first born result the peak is slightly shifted for the increasing ejected angle $\left(\theta_{1}\right)$.

From Fig-4-9 we compared our present result with second born 2P metastable state hydrogen atoms result [39] and the second born $2 \mathrm{~S}$-state [12] result. We also include here the present first born result [42].

Here in Fig. 4 the picture shows a good agreement of our present result with $2 \mathrm{~S}$-state metastable result. The present TDCS curve display one fall and one peak in recoil region and in a similar way it shows also a peak in the binary region while for 2P state metastable curve the TDCS exhibit one prominent peak in recoil region and one is in binary region.

In Fig. 5 our present curve shows almost a similar result with $2 \mathrm{P}$ metastable state whereas the $2 \mathrm{~S}$-state curve shows a prominent peak in the binary peak region. And in Fig. 6 as we increase our scattering angle $\theta_{2}$ the compared four TDCS curves express almost a similar position with our present result.

In Fig. 7 our present TDCS curve shows a very interesting result. It exhibits two falls in recoil region and two peaks in binary region. The result shows a bit different from $2 \mathrm{P}$ - state result [39].

In the Fig. 8 the present TDCS curve displays a good similarity with $2 \mathrm{P}$ state result in recoil region and at the same time the result shows a good agreement with $2 \mathrm{~S}$ - state result in binary region.

For this similar segment the last Fig. 9 is for the highest scattering angle our present curve shows almost a similar position with our first born result and $2 \mathrm{P}$ metastable state result both in recoil region and binary region. But the present peak value in the recoil region is slightly smaller than the hydrogenic metastable $2 \mathrm{~S}$ - state [12] result.

In Fig. 10 we calculated the first born TDCS for the ejection of $5 \mathrm{eV}$ electron energy in the direction of the momentum transfer in the ionization of different kinematic state by $250 \mathrm{eV}$ incident energy. The present peak value shows a perfectly similar but the recoil positon magnitude is slightly greater than the compared results of Vučič et al. [19].

In equation (7), the term $\phi_{\bar{p}_{1}}^{(-)}\left(\bar{r}_{1}\right) e^{i, \bar{p}_{2}, \bar{r}_{2}}$ is the first born amplitude where the scattered electrons are described by a plane wave whereas the ejected electrons are described by a coulomb wave. The term $\phi_{\bar{p}_{2}}^{(-)}\left(\bar{r}_{2}\right) e^{i, \bar{p}_{1}, \bar{r}_{1}}$ is equal to the first born amplitude excluding the fact that the role of the electrons and projectiles are interchanged. The term $\phi_{P}^{(-)}(\bar{r}) e^{i \bar{P} \cdot \bar{r}}$ represents the projectile electron interaction which is preserved in the final channel. The term $e^{i, \bar{p}_{1}, \bar{r}_{1}+i, \bar{p}_{2}, \bar{r}_{2}}$ signifies the two plane waves for the ejected electron and scattered particle.

The above experimental graph gives us a clear view of peaks both in recoil region and binary region. We can conclude that the present peak values gives us a good agreement with our compared experimental results especially with the recent works of metastable 2P-state and 3S-state hydrogen atoms. In Our present study the measurements of peak values gives us the encouragement of further research in this field of interest.

\section{Conclusions}

Our present calculation exposes a thinkable additional structures of the cross-section curves for small momentum transfer in the ionization of the hydrogen atoms in the metastable 3S-state by $250 \mathrm{eV}$ electron impact. When we use the full wave function of multiple scattering theory [11] the present results give us a perfect qualitative agreement with the compared experimental data [5] and theoretical results of hydrogenic ground state BBK results [31] and second born results [25]. It is also noted that at a highest scattering angle our present result shows a good agreement with the hydrogenic 2S-state [12] and 2P-state [39] results. Present first born result make a good fit when we compared our result with Vučič et al. [19]. This result gives us a wider scope of further experimental works on metastable 3S-state hydrogen atoms. Due to unavailable experimental results on hydrogenic 3S-state ionization process, we cannot compare our result with such experimental work. There needs some experimental works in this field for further judgment of our work.

\section{Acknowledgement}

The present computational works have been performed in the Simulation Lab of the Department of Mathematics, Chittagong University of Engineering and Technology, Chittagong 4349, Bangladesh.

\section{References}

[1] Bethe H. A Zur Theorie des Durchgangs schneller Korpuskularstrahlen durch Materie. Asymmetric Ann Phys 1930; 5:325. Handbuch der Physik Ed. A Smekal Aufbau Der Zusammenhangenden Materie. 1933: 24:273 
[2] Weigold E, Noble CJ, Hood ST, Fuss I, Electron impact ionization of atomic hydrogen: experimental and theoretical (e, 2e) differential cross section. J Phys B At Mol Opt Phys 1979: 12:291

[3] Lahmann B McCarthy IE, Stelbovics AT, Weigold E, Electronimpact ionization of atomic hydrogen: comparison of asymmetric (e, 2e) measurements with theories. Phys Rev A 1984: 30: 758

[4] Ehrhardt H, Knoth G, Schlemmer P, Jung K, Absolute H (e, 2e)p cross section measurements: comparison with first and second order theory. Phys Lett A 1985: 110: 92

[5] Ehrhardt H, Knoth G, Schlemmer P, Jung K.Differential cross sections of direct single electron impact ionization. Z Phys D 1986: $1: 3$

[6] Ehrhardt H. Jung, K. Knoth, G. Schlemmer P. Differential cross sections of direct single electron impact ionization. New York:Plenum: 1997.p.1-10

[7] Byron WF. Joachain JC. Piraux B. Triple differential cross sections for the ionization of atomic hydrogen by fast electrons: a second born treatment. J phys B At Mol Opt Phys 1980: 13

[8] Byron Jr JW. Joachain CJ. Piraux B. Theory of coplanar asymmetric (e, 2e) reactions in helium. J Phys B At Mol Opt Phys 1886; 19:1201

[9] Byron FW, Joachain CJ, Piraux B. Triple differential cross sections for the ionization of atomic hydrogen by fast electrons: a second born treatment. J Phys B At Mol Opt Phys $1985 ; 18: 3203$

[10] Das JN. Momentum-space analysis of scattering states with possible application to atomic ionization. Phys Rev A 1990; 42: 1376

[11] Das JN. Seal S. Electron-hydrogen-atom ionization collision at intermediate $\left(2 l_{0}-20 l_{0}\right)$ and high $\left(\geq 20 l_{0}\right)$ energies. Phys Rev A 1993; 47: 2978

[12] Das JN. Seal S. Symmetric scattering in $e^{H}-H$ ionization collisions. Pramana J Phys 1993; 40: 253

[13] Das JN. Dhar S. Energy spectrum of ejected electrons in ionization of hydrogen atoms by electrons. Pramana J Phys 1999; 53: 869

[14] Jones S. Madison DH. Ionization of hydrogen atoms by fast electrons. Phys Rev A 2000; 62: 042701

[15] Jones S. Madison DH. Scaling behavior of the fully differential cross section for ionization of hydrogen atoms by the impact of fast elementary charged particles. Phys Rev A 2002; 65: 052727

[16] Li Sm. Berakdar J. Zhang ST. Chen J. Laser assisted (e, 2e) reaction in one electron atoms and ions. J Phys B At Mol Opt Phys 2005; 38: 1291

[17] Das JN. Dhar S. Energy spectrum of scattered electrons in Kshell ionization of medium to heavy atoms by relativistic electrons. J Phys B At Mol Opt Phys 1998; 31: 2355

[18] Bransden BH.Joachain CJ.Physics of atoms and molecules. $2^{\text {nd }}$ Ed. New York: Pearson Education; 1983

[19] Vuči ič S, Potvliege RM, Joachain CJ. Second born tripledifferential cross sections for the coplanar asymmetric ionization of $\mathrm{H} \quad(2 \mathrm{~S})$ by fast electrons. Phys Rev A $1987 ; 35: 1446$

[20] Ray H, Roy AC. Triply differential cross sections for the coplanar asymmetric ionization of $\mathrm{H}(2 \mathrm{~S})$ by fast electrons. J Phys B At Mol Opt Phys 1988; 21:3243

[21] Hafid H. Joulakian B. Dal Cappelo C. Theoretical Study of the differential cross section of the ionization of hydrogen (2S) by electron impact. J Phys B At Mol Opt Phys 1993; 26: 3415

[22] Das JN. Dhar S. Symmetric scattering in electron and positron impact ionization of metastable 2S-state hydrogen atoms. Pramana J Phys 1996; 47:263

[23] Dhar S. Electron impact ionization of metastable 2S-state Hydrogen atoms Aust J Phys 1996; 49: 937

[24] Das JN. Dhar S. Calculation of triple differential cross-sections of K-shell ionization of medium-heavy atoms by electrons for symmetric geometry. Pramana J Phys 1998; 51: 751

[25] Dal Cappelo C. Haddadou A. Menas F. Roy AC. The second born approximation fr the single and double ionization of atoms by electrons and positrons. J Phys B At Mol Opt Phys 2011; 44: 015204

[26] Berakdar J. Engelns A. Klar H. Oriented and Aligned twoelectron continue. J Phys B At Mol Opt Phys 1996; 29: 1109

[27] Karnali MZM, Ratnavelu K, Zhou Y. Electron impact excitation of $2 \mathrm{P}$ and $3 \mathrm{P}$ states of hydrogen at intermediate energies. Eur Phys J D 2008; 46: 267-79

[28] Qi YY. Ning LN. Wang JG, Qu YZ. Plasma effect on fastelectron-impact-ionization from $2 \mathrm{P}$ state of hydrogen-like ions. Phys Plasmas 2013; 20: 123301

[29] Brauner M. Briggs J S and Klar H 1986 J. Phys. B: At. Phys. 19 L 325

[30] Brauner M. and Briggs J S 1991 J.Phys. B: At.Mol.Opt. Phys. 242227

[31] Brauner M. Briggs J S and Klar H. Triply-Differential cross sections for ionization of hydrogen atoms by electrons and positrons. J. Phys. B: At. Mol. Opt. Phys. 1989; 22: 2265

[32] Ren X. Dorn A and Ullrich J 2008 Phys, Rev. Lett. 101

[33] Defrance P. Clays W. Cornet A. Poulaert G. J Phys B At. Mol Phys 1981: 14: 11

[34] Dixon Aj. Harrison MFA, Smith ACH. J Phys B At. Mol Phys 1976: 9: 2617

[35] Dhar S. Alam MR.Computation of triple differential crosssections with the inclusion of exchange effects in atomic Kshell ionization by relativistic electrons for symmetric geometry. Pramana J Phys 2007; 69: 384

[36] Dhar S. The energy spectrum of scattered particles in the Kshell ionization of medium heavy atoms by relativistic electrons and positrons with exchange effects. J Phys B At Mol Phys 2008; 41: 155204

[37] Amaldi Jr U, Egidi A, Marconero R, Pizzella G. Rev. Sci. Instr. 1969; 40: 1001

[38] Lewis RR. Potential scattering of high-energy electrons in second born approximation. Phys Rev 1956; 102: 537 
[39] Dhar S., Nahar N. Electron impact ionization of metastable $2 \mathrm{P}$-state hydrogen atoms in the coplanar geometry. Results in Phys.2015; 5: 3-8

[40] Dhar S., Nahar N. Energy spectrum of ejected electrons of H (2P) ionization by electrons in coplanar asymmetric geometry. J. Ajmp. 2015; 4 (3): 132

[41] Dhar S., Nahar N. Theoretical analysis of non-relativistic first order born approximation for ionization of $\mathrm{H}(2 \mathrm{P})$ by electron impact
[42] Dhar S., Noor T. Chowdhury FS. Electron impact ionization of metastable 3S-state hydrogen atoms by electrons in coplanar geometry. J. Ajmp. 2015; 4 (6): 361-366

[43] Dhar S. Akter S. Nahar N.The first born triple differential cross sections for ionization of $\mathrm{H}(3 \mathrm{P})$ by electron impact in the asymmetric coplanar geometry. J Ojmp. 2016; 6: 15-23 